Article

\title{
Cmfhp Gene Mediates Fruiting Body Development and Carotenoid Production in Cordyceps militaris
}

\author{
Hai-Wei Lou ${ }^{1,2}{ }^{\circledR}$, Yu Zhao ${ }^{2}$, Bai-Xiong Chen ${ }^{1}$, Ying-Hao Yu ${ }^{1}$, Hong-Biao Tang ${ }^{1}$, Zhi-Wei Ye ${ }^{1}$, \\ Jun-Fang Lin ${ }^{1,3, *(D)}$ and Li-Qiong Guo ${ }^{1,3, *}$ \\ 1 Department of Bioengineering, College of Food Science, South China Agricultural University, \\ Guangzhou 510640, China; superharry@163.com (H.-W.L.); bxiong@foxmail.com (B.-X.C.); \\ yuyinghao6@163.com (Y.-H.Y.); hbtang25@163.com (H.-B.T.); zhwye@scau.edu.cn (Z.-W.Y.) \\ 2 College of Food Science and Technology, Henan University of Technology, Zhengzhou 450001, China; \\ zyzhaoyu@yeah.net \\ 3 Research Center for Micro-Ecological Agent Engineering and Technology of Guangdong Province, \\ Guangzhou 510640, China \\ * Correspondence: linjf@scau.edu.cn (J.-F.L.); guolq@scau.edu.cn (L.-Q.G.)
}

Received: 14 January 2020; Accepted: 3 March 2020; Published: 6 March 2020

\begin{abstract}
Cordyceps militaris fruiting bodies contain a variety of bioactive components that are beneficial to the human body. However, the low yield of fruiting bodies and the low carotenoid content in C. militaris have seriously hindered the development of the C. militaris industry. To elucidate the developmental mechanism of the fruiting bodies of $C$. militaris and the biosynthesis mechanism of carotenoids, the function of the flavohemoprotein-like $C m f h p$ gene of $C$. militaris was identified for the first time. The Cmfhp gene was knocked out by the split-marker method, and the targeted gene deletion mutant $\Delta C m f h p$ was obtained. An increased nitric oxide (NO) content, no fruiting body production, decreased carotenoid content, and reduced conidial production were found in the mutant $\Delta C \mathrm{mfhp}$. These characteristics were restored when the Cmfhp gene expression cassette was complemented into the $\Delta C m f h p$ strain by the Agrobacterium tumefaciens-mediated transformation method. Nonetheless, the $C m f h p$ gene had no significant effect on the mycelial growth rate of $C$. militaris. These results indicated that the Cmfhp gene regulated the biosynthesis of NO and carotenoids, the development of fruiting bodies, and the formation of conidia. These findings potentially pave the way to reveal the developmental mechanism of fruiting bodies and the biosynthesis mechanism of carotenoids in C. militaris.
\end{abstract}

Keywords: Cordyceps militaris; fruiting body; carotenoid; flavohemoprotein; nitric oxide; gene function

\section{Introduction}

Cordyceps militaris is a well-known medicinal and edible filamentous fungus. It has been widely used in many countries because it contains many kinds of bioactive components (such as cordycepin, cordycepic acid, polysaccharides, carotenoids, ergosterol, etc.) [1-4]. These bioactive components endow C. militaris with anticancer, antitumor, anti-inflammatory, and antioxidant activities [5-7]. In recent years, novel carotenoids with good water solubility and pentostatin with anticancer activity have been found in C. militaris, and these findings have increased the market demand for C. militaris every year $[8,9]$.

Natural carotenoids play an important role in human health and food coloring. It was suggested that the carotenoid content should be considered as the quality standard of the commercial products of C. militaris [10]. C. militaris, a potential natural carotenoid resource, cannot meet the market demand because of its low carotenoid content [11,12]. A good way to improve the carotenoid content of 
C. militaris is by genetic engineering. However, there are few reports on carotenoid biosynthesis genes [13]. Therefore, it is necessary to study the genes involved in carotenoid biosynthesis in C. militaris.

The main consumer's demand for C. militaris is its fruiting bodies. Hence, scientists have been working to increase the yield of $C$. militaris fruiting bodies [14,15]. However, the developmental mechanism of $C$. militaris fruiting bodies remains unclear. In addition, when $C$. militaris is preserved for a long time or subcultured for many times, its ability to produce fruiting bodies will weaken, or even lose the ability to produce fruiting bodies, which is the degeneration of $C$. militaris $[16,17]$. The degeneration of $C$. militaris often results in a decrease in the yield of fruiting bodies or in the absence of fruiting bodies [16,17]. Therefore, studying the genes involved in the formation of $C$. militaris fruiting bodies will be helpful to improve the yield of fruiting bodies and will reveal the molecular mechanism of $C$. militaris degeneration.

Light is an essential factor for pigment formation and fruiting body development in C. militaris [18]. In our previous experiments, we compared and analyzed the differences between the transcriptome of $C$. militaris mycelia CM10_D cultured under dark conditions and the transcriptome of $C$. militaris mycelia CM10_L cultured under light conditions [19]. We found that the expression level of the Cmfhp gene (Gene ID: 18167139) in mycelia CM10_L was significantly higher than that in mycelia CM10_D. The annotation results of the KEGG Orthology $(\mathrm{KO})$ database indicated that the expression product of the Cmfhp gene was nitric oxide (NO) dioxygenase. The annotation results of the NCBI non-redundant (NR) protein database showed that the expression product of the Cmfhp gene was flavohemoprotein (NCBI accession number: XP_006670328.1). Both annotations indicated that the function of the Cmfhp gene was to catalyze the oxidation of $\mathrm{NO}$ to nitrate. $\mathrm{NO}$ is a signal transduction molecule that plays a variety of important roles in fungi [20]. Previous studies demonstrated that NO regulated the formation of fungal conidia, the growth of mycelia, and the formation of fruiting bodies [21]. In addition, NO is a toxic molecule, and the flavohemoprotein can protect cells from NO toxicity [22]. Therefore, the aim of this study was to study the effects of the Cmfhp gene on the NO and carotenoid contents, fruiting body development, and conidial formation of $C$. militaris by gene knockout and gene complementation. This study lays a substantial foundation for revealing the developmental mechanism of fruiting bodies and the biosynthetic pathway of $C$. militaris carotenoids.

\section{Materials and Methods}

\subsection{Strains and Plasmids}

The C. militaris strain CM10 (GIM5.271) was maintained on potato dextrose agar (PDA) at $4{ }^{\circ} \mathrm{C}$ as a stock. Escherichia coli DH5 $\alpha$ carried a plasmid pCAMBIA0390-Bar-KOfhp (Figure S1). Agrobacterium tumefaciens AGL-1 carried a plasmid pCAMBIA0390-Ben-Comfhp containing the Cmfhp gene (Figure S2).

\subsection{Disruption of the Cmfhp Gene in C. militaris}

The Cmfhp gene was knocked out by the split-marker method as previously described [23]. The split-marker deletion cassettes were prepared by PCR using the plasmid pCAMBIA0390-Bar-KOfhp as a DNA template. The $5^{\prime}$ split-marker fragment (2292 bp) was amplified with the primers KOfhpU-F and KOfhpU-R. The $3^{\prime}$ split-marker fragment (2801 bp) was amplified with the primers KOfhpD-F and KOfhpD-R (Figure S3 and Table S1). In addition, mononuclear protoplasts of $C$. militaris were prepared by the previously described method [24]. Finally, the split-marker fragments were transformed into mononuclear protoplasts by the previously described transformation method [23]. Colonies that could grow on resistant PDA containing glufosinate ammonium $(300 \mu \mathrm{g} / \mathrm{mL})$ were considered to be putative transformants. The $C m f h p$ gene deletion mutant $(\Delta C m f h p$ ) was verified by PCR and Southern blot hybridization according to previously reported methods [23]. PCR products were sequenced at Majorbio BioTech Co. (Guangzhou, China) to verify the sequences. 
The deletion of the Cmfhp gene was further confirmed by quantitative real-time PCR (qRT-PCR) using the tef1 gene (GenBank: DQ070019) as the internal control gene [25]. Primers tef1-F and tef1-R (for detecting the tef1 gene) and primers Qfhp-F and Qfhp-R (for detecting the Cmfhp gene) are listed in Table S1. All qRT-PCR was carried out according to previously described methods [26]. The relative expression level of the Cmfhp gene was calculated relative to tef1 expression using the $2^{-\Delta \Delta C T}$ method [27].

\subsection{Complementation of the Cmfhp Disruption Mutant}

The A. tumefaciens AGL1-pCAMBIA0390-Ben-Comfhp and conidia of the mutant $\Delta C m f h p$ were co-cultured to achieve complementation of the Cmfhp gene by the A. tumefaciens-mediated transformation (ATMT) method [28]. Colonies that could grow on resistant PDA containing $3 \mu \mathrm{g} / \mathrm{mL}$ of benomyl were considered to be putative transformants. The successful complementary transformants $(\Delta C m f h p-c)$ were verified by PCR and qRT-PCR.

\subsection{Determination of the NO Content}

The NO content in C. militaris was determined using an NO assay kit (Nanjing Jiancheng Bioengineering Institute, Nanjing, China) according to the manufacturer's instructions. One gram of fresh C. militaris mycelia was ground in $5 \mathrm{~mL}$ of $40 \mathrm{mM}$ 4-(2-hydroxyethyl)-1-piperazineethanesulfonic acid ( $\mathrm{pH} 7.2$ ) to assess the NO content. The homogenate was centrifuged at $14,000 \times g$ for $10 \mathrm{~min}$. The supernatant was used to measure NO [29]. Three biological replicate experiments were performed on each strain of $C$. militaris.

\subsection{Cultivation of C. militaris Fruiting Bodies}

The fruiting bodies of all C. militaris strains (CM10, $\Delta C m f h p$, and $\Delta C m f h p-c)$ were cultured on rice medium according to a previously described method [14].

\subsection{Determination of Carotenoid Content}

All C. militaris strains (CM10, $\Delta C m f h p$, and $\Delta C m f h p-c)$ were cultured on PDA for 3 weeks under dark conditions and then 1 week under light conditions. The $C$. militaris mycelia were collected and vacuum freeze-dried. The dried mycelia were used for the determination of carotenoid content according to previously reported methods $[10,12]$.

\subsection{Growth Rate and Conidial Production}

The strains of $C$. militaris (CM10, $\Delta C m f h p$, and $\Delta C m f h p-c$ ) were inoculated on PDA at $25^{\circ} \mathrm{C}$ for 3 weeks before use. A 5-mm disk was punched with a sterilized cutter from the prepared PDA inoculum and transferred to a fresh PDA plate. PDA plates with C. militaris inoculum were cultured in the dark at $25^{\circ} \mathrm{C}$. The growth rate was determined by measuring the colony diameter after 3 weeks of incubation [12]. Then, the colonies on PDA medium that had been cultured for 3 weeks were used to determine the production of conidia. Mycelia were scraped from the PDA plates and resuspended in $10 \mathrm{~mL}$ of a Tween 80 solution $(20 \%, w / v)$. After filtration, the conidial suspensions were counted using a hemocytometer under a microscope $[18,30]$.

\subsection{Statistical Analysis}

All experiments were carried out in triplicate. Data were analyzed by SPSS 22.0 software (SPSS Inc., Chicago, IL, USA). The values are shown as the mean \pm standard error. $p$-values less than 0.05 were considered significant. 


\section{Results}

\subsection{Disruption and Complementation of the Cmfhp Gene}

Using the plasmid pCAMBIA0390-Bar-KOfhp as a DNA template, the $5^{\prime}$ split-marker fragment and the $3^{\prime}$ split-marker fragment were prepared by PCR amplification (Figure 1a,b). C. militaris protoplasts were prepared from mycelia (Figure 1c). Then, $5^{\prime}$ split-marker fragment and the $3^{\prime}$ split-marker fragment were co-transformed into C. militaris protoplasts. The results of PCR analysis showed that the bar gene was successfully integrated into the C. militaris genome and that the Cmfhp gene was knocked out (Figure 1d). The results of hybridization with the bar probe suggested that there were three hybridization bands in the PCR-positive mutant of lane 19, one hybridization band in the PCR-positive mutant of lane 20, and no hybridization band in wild-type C. militaris (Figure 1e).
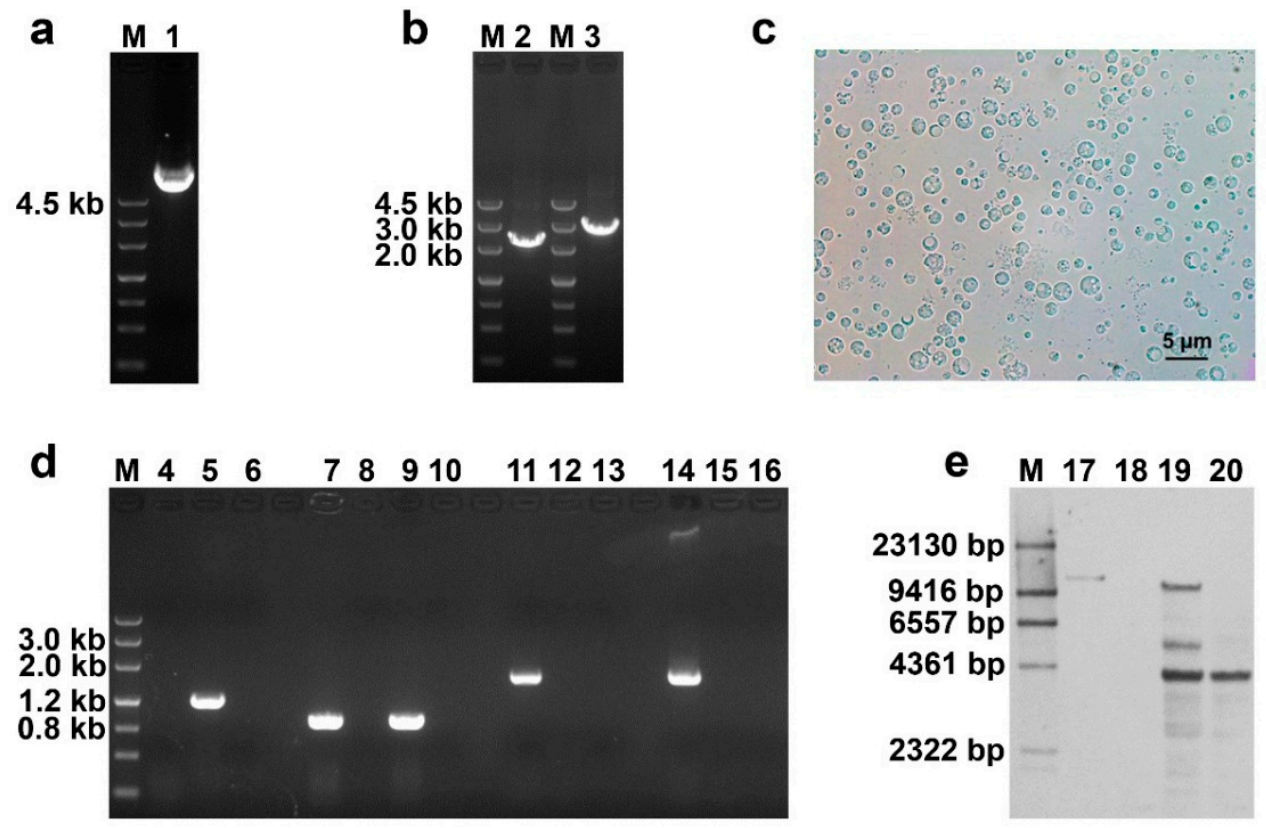

Figure 1. Knockout of the Cmfhp gene. (a) Extraction of the plasmid pCAMBIA0390-Bar-KOfhp (M: DNA marker; lane 1: plasmid pCAMBIA0390-Bar-KOfhp). (b) Preparation of split-marker fragments (M: DNA marker; lane 2: 5' split-marker fragment; lane 3: 3' split-marker fragment). (c) Preparation of Cordyceps militaris protoplasts. (d) PCR analysis of the Cmfhp gene deletion. Four DNA templates (lanes 4, 7, 11, and 14: genome of the mutant; lanes 5, 8, 12, and 15: genome of wild-type C. militaris; lane 9: plasmid pCAMBIA0390-Bar; lanes 6, 10, 13, and 16: $\mathrm{ddH}_{2} \mathrm{O}$ ) were used as DNA templates. The 1196 bp fragment of the Cmfhp gene was amplified (lanes 4-6). The $890 \mathrm{bp}$ fragment of the bar cassette was amplified (lanes 7-10). The upstream flanking sequence (1852 bp) was amplified (lanes 11-13). The downstream flanking sequence (2047 bp) was amplified (lanes 14-16). (e) Digested DNA was hybridized with a bar probe for Southern blot analysis of PCR-positive mutants (M: DIG-labeled marker; lane 17: EcoRV-digested plasmid pCAMBIA0390-Bar-KOFhp, 11,592 bp; lane 18: PstI-digested genome of wild-type C. militaris; lanes 19-20: PstI-digested genome of PCR-positive mutants).

The mutant corresponding to lane 20 was used for qRT-PCR analysis. The qRT-PCR results demonstrated that the expression of the Cmfhp gene was not detected in the mutant $\Delta C m f h p$ (Figure 2). Based on these results, the Cmfhp gene was successfully knocked out, and the $\Delta C m f h p$ strain was used for subsequent experiments. 


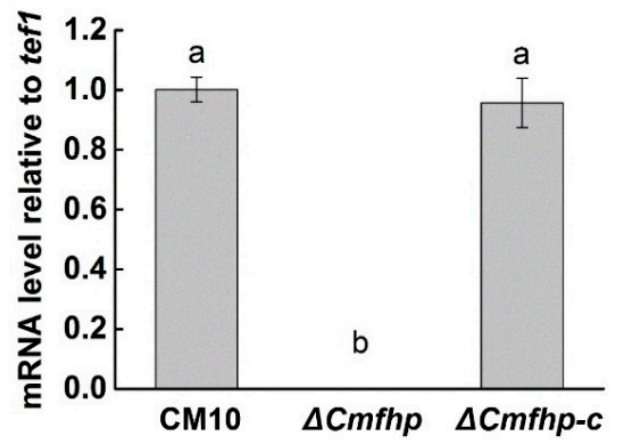

Figure 2. qRT-PCR analysis of the relative expression of the Cmfhp gene in C. militaris transformants using wild-type $C$. militaris CM10 as a control. Different letters $(a, b)$ indicate significant differences in the relative expression levels of the Cmfhp gene.

The results of PCR amplification of the ben gene and the Cmfhp gene in the complementary transformants are shown in Figure 3. The sequencing results of the PCR products confirmed that the ben gene and the Cmfhp gene were successfully integrated into the genome of the $\Delta C m f h p$ mutant. The results of qRT-PCR analysis indicated that there was no significant difference in the relative expression level of the Cmfhp gene between the wild-type C. militaris CM10 and the complementary transformant, which indicated that the Cmfhp gene was successfully complemented to the $\Delta C m f h p$ mutant and could be expressed in the complementary transformant $(\Delta C m f h p-c)$ (Figure 2).
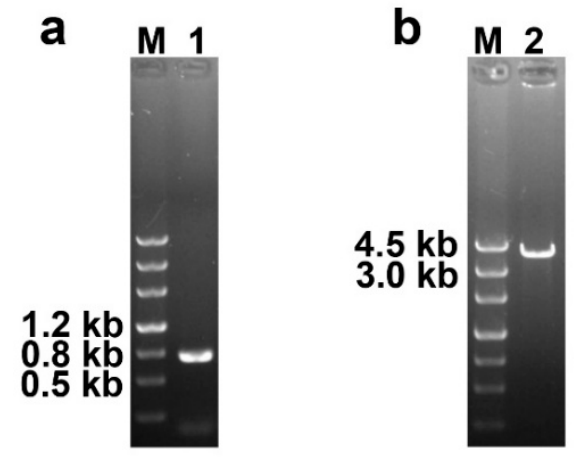

Figure 3. PCR analysis of the complementation of the Cmfhp gene. (a) PCR amplification of the ben gene in complementary transformants (M: DNA marker; lane 1: $754 \mathrm{bp}$ fragment of the ben cassette). (b) PCR amplification of the Cmfhp gene in complementary transformants (M: DNA marker; lane 2: the Cmfhp cassette).

\subsection{Effect of the Cmfhp Gene on the NO Content in C. militaris}

Compared with wild-type $C$. militaris $\mathrm{CM} 10, C$. militaris $\Delta C \mathrm{mfh} p$ contained more NO, which might be due to the deletion of the Cmfhp gene. Moreover, the NO content in the complementary strain $\Delta C m f h p-c$ was restored to the levels in wild-type C. militaris CM10 (Figure 4). Therefore, the expression product of the Cmfhp gene could metabolize NO in C. militaris and reduce the content of NO in C. militaris, and these effects were consistent with the previously reported function of flavohemoprotein [31].

\subsection{Effect of the Cmfhp Gene on the Development of C. militaris Fruiting Bodies}

The fruiting bodies of wild-type C. militaris CM10 cultured on rice medium were irregular lumps in shape and were orange in color (Figure 5a). However, C. militaris $\Delta C m f h p$ cultured on rice medium did not produce fruiting bodies (Figure $5 b$ ). When the $C m f h p$ gene was complemented to the mutant $\Delta C m f h p$, the complementary strain $\Delta C m f h p-c$ had a restored ability to produce fruiting bodies. 
Moreover, the fruiting bodies of the complementary strain $\Delta C m f h p-c$ were still irregular lumps in shape and were orange in color (Figure $5 \mathrm{c}$ ). These results suggested that the Cmfhp gene was involved in the development of C. militaris fruiting bodies.

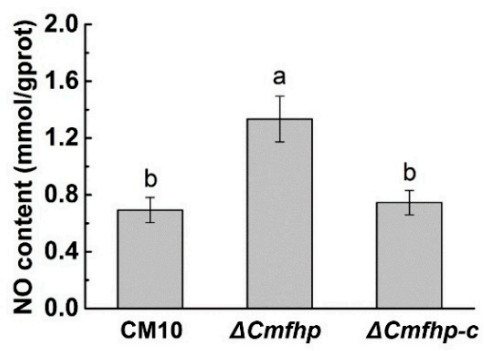

Figure 4. Analysis of the nitric oxide (NO) content in C. militaris. Different letters $(a, b)$ indicate significant differences in the $\mathrm{NO}$ content.

a

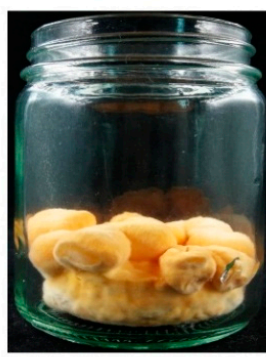

d
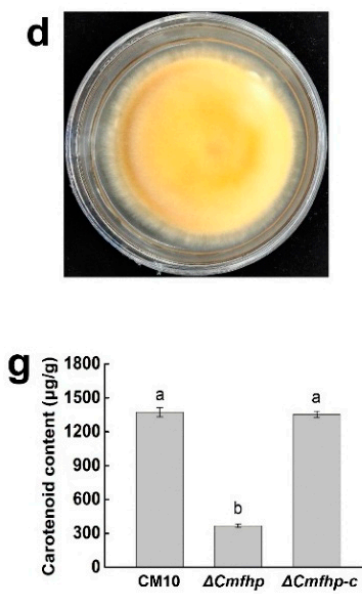

b

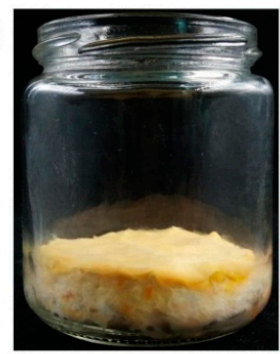

e

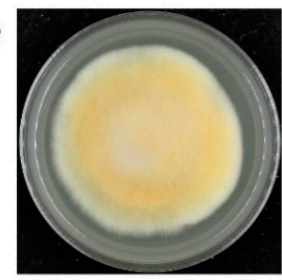

c
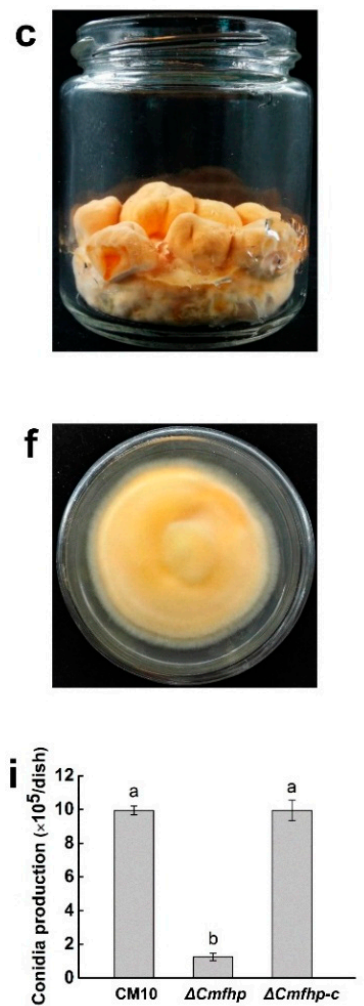

Figure 5. Phenotypic analysis of C. militaris. (a) C. militaris CM10 cultured on rice medium. (b) C. militaris $\Delta$ Cmfhp cultured on rice medium. (c) C. militaris $\Delta C m f h p-c$ cultured on rice medium. (d) C. militaris CM10 cultured on potato dextrose agar (PDA) medium. (e) C. militaris $\Delta$ Cmfhp cultured on PDA medium. (f) C. militaris $\triangle C m f h p-c$ cultured on PDA medium. (g) Analysis of the carotenoid content in C. militaris. (h) Analysis of the growth rate of $C$. militaris. (i) Analysis of conidial production of $C$. militaris. Different letters $(a, b)$ indicate significant differences.

\subsection{Effect of the Cmfhp Gene on the Carotenoid Content of C. militaris}

The C. militaris strains CM10, $\Delta C m f h p$, and $\Delta C m f h p-c$ were all cultured on PDA medium (Figure $5 \mathrm{~d}-\mathrm{f}$ ). The $C$. militaris strains CM10 and $\Delta C m f h p-c$ were all orange in color; however, the color of the C. militaris strain $\Delta C m f h p$ was light yellow. These results implied that the Cmfhp gene regulated the production of $C$. militaris pigments. In addition, the analysis of carotenoid content showed that the carotenoid content of $C$. militaris CM10 was not significantly different from that of C. militaris $\Delta C m f h p-\mathcal{C}$ 
but was significantly higher than that of $C$. militaris $\Delta C m f h p$ (Figure 5g). Therefore, we believe that the Cmfhp gene regulated the production of carotenoids in C. militaris.

\subsection{Effect of the Cmfhp Gene on the Growth Rate and Conidial Production of C. militaris}

The analysis results of the mycelial growth rate showed that there was no significant difference in the growth rates of the C. militaris strains CM10, $\Delta C m f h p$, and $\Delta C m f h p-c$ (Figure $5 \mathrm{~h}$ ). This indicated that the Cmfhp gene had no significant effect on the growth of $C$. militaris. However, the conidial production of the mutant $\Delta C m f h p$ was significantly lower than that of the wild-type C. militaris, and there was no significant difference in the conidial production between the wild-type strain CM10 and the complementary strain $\Delta C m f h p-c$ (Figure 5i). These results demonstrated that the Cmfhp gene significantly affected the conidial production of $C$. militaris.

\section{Discussion}

Light is a necessary condition for the production of $C$. militaris fruiting bodies and pigments [18]. The Cmfhp gene of $C$. militaris was significantly upregulated after being treated with light. In the present study, the function of the Cmfhp gene in C. militaris was studied for the first time by gene knockout and gene complementation. We found that the Cmfhp gene not only affected the formation of C. militaris fruiting bodies and conidia but also regulated the production of $\mathrm{NO}$ and carotenoids. However, the $C m f h p$ gene had no significant effect on the growth rate of $C$. militaris mycelia.

Flavohemoprotein, a NO dioxygenase, is capable of oxidizing NO to nitrate using oxygen [22]. This enzymatic conversion thus protects the cell from toxic NO and from other damaging NO-derived reactive nitrogen species [22]. The deletion of the Cmfhp gene led to an accumulation of more NO in the $\Delta C m f h p$ mutant, which might be due to the absence of the flavohemoprotein (encoded by the Cmfhp gene) that could catalyze the oxidation of $\mathrm{NO}$ to nitrate. In addition, $\mathrm{NO}$ produced at sufficient levels directly or indirectly damages critical cell processes [32]. Hence, excessive NO might be toxic to the development and metabolism of $C$. militaris.

The consumer's demand for C. militaris is mainly for its fruiting bodies. However, the developmental mechanism of C. militaris fruiting bodies is poorly understood. Although C. militaris is a heterothallic ascomycetous fungus, it can still produce fruiting bodies without an opposite mating-type partner [33]. Previous studies have also shown that the mating-type is not the decisive factor for the production of $C$. militaris fruiting bodies [34,35]. Therefore, it is necessary to study the key genes involved in the formation of $C$. militaris fruiting bodies. In this study, the wild-type C. militaris CM10 with a single mating-type gene (MAT 1-1) can stably produce irregular lumpy fruiting bodies. The phenotypic analysis of the fruiting bodies of $C$. militaris strains (CM10, $\Delta C m f h p$, and $\Delta C m f h p-c$ ) revealed that the $C m f h p$ gene was a key gene affecting the formation of $C$. militaris fruiting bodies. It has been previously reported that NO regulates the formation of fungal fruiting bodies [21]. Therefore, we believe that the loss of the ability to produce fruiting bodies of the $\Delta \mathrm{Cmfhp}$ strain may be due to its high NO content.

Carotenoids are secondary metabolites produced by C. militaris cultured under the light. However, the biosynthetic pathway of $C$. militaris carotenoids is still unknown, and there are few reports on the genes involved in the biosynthesis of $C$. militaris carotenoids. A putative carotenoid biosynthetic pathway for $C$. militaris was proposed, but 11 genes involved in this putative pathway were not significantly differentially expressed between the C. militaris strain 498 and the C. militaris strain 505 after light irradiation [13]. Although three types of geranylgeranyl diphosphate synthase genes in C. militaris were cloned, their functions have yet to be identified [36]. It is noteworthy that the other two key enzymes (phytoene synthetase and phytoene dehydrogenase) were not found in the genome of C. militaris $[13,18]$. The induction of carotenoids was completely different between C. militaris and Neurospora crassa $[13,18]$. Therefore, it is a great challenge to characterize the carotenoid biosynthetic pathway in C. militaris. In this study, the Cmfhp gene was identified to have a significant effect on the biosynthesis of carotenoids in C. militaris. The carotenoid content of the $\Delta \mathrm{Cmfhp}$ strain was lower 
than that of wild-type C. militaris CM10, while the NO content of the $\Delta C m f h p$ strain was significantly higher than that of wild-type $C$. militaris CM10. It has been reported that NO could downregulate the synthesis of carotenoids in Chlamydomonas reinhardtii [37]. Therefore, we believed that the excessive NO in the $\Delta C m f h p$ strain inhibited the biosynthesis of carotenoids.

NO inhibited the mycelial growth of Aspergillus niger, Monilinia fructicola, Penicillium italicum, and $N$. crassa [21,38]. In the present study, although the NO content in the $\Delta C m f h p$ strain was higher than that in wild-type C. militaris CM10, there was no significant difference in their growth rate. This might be due to the different tolerance thresholds of different fungi to $\mathrm{NO}$, and the NO content in the $\Delta \mathrm{Cmfhp}$ strain might not reach the level that inhibits the growth of $C$. militaris mycelia. It has also been reported that NO inhibited the conidial production of N. crassa [21,39]. In this study, the conidial production in C. militaris (CM10, $\Delta C m f h p$, and $\Delta C m f h p-c$ ) was negatively correlated with the content of NO. This suggests that the conidial production of $C$. militaris may be inhibited by NO.

Flavohemoprotein could detoxify NO in A. fumigatus [31]. However, the detoxification mechanism of NO in C. militaris is still unknown. Therefore, the following aspects will need to be studied: (1) elucidating the formation mechanism of NO in C. militaris; (2) revealing the expression mechanism of the Cmfhp gene in C. militaris; (3) clarifying the NO detoxification mechanism of the Cmfhp gene; (4) elucidating the regulation mechanism of $\mathrm{NO}$ on the development of fruiting bodies and the metabolism of bioactive ingredients in C. militaris. The identification of the function of the Cmfhp gene in this study will also help to reveal the developmental mechanism of fruiting bodies and the biosynthesis mechanism of carotenoids in C. militaris.

\section{Conclusions}

The function of the Cmfhp gene in C. militaris was identified by gene knockout and gene complementation for the first time, and the target gene deletion mutant $\Delta C m f h p$ and the target gene complementary strain $\Delta C m f h p-c$ were obtained. The deletion of the Cmfhp gene resulted in an increase in NO content, the loss of the ability to produce fruiting bodies, a decrease in the carotenoid content, and a reduction in conidial production in the $\Delta C m f h p$ mutant. However, the deletion of the Cmfhp gene had no significant effect on the mycelial growth rate of $C$. militaris. The identification of the function of the Cmfhp gene will be helpful to reveal the developmental mechanism of C. militaris fruiting bodies and the metabolic regulation mechanism of carotenoids in C. militaris.

Supplementary Materials: The following are available online at http://www.mdpi.com/2218-273X/10/3/410/ s1, Table S1: Oligonucleotide primer sequences used in this study, Figure S1: Construction of the vector pCAMBIA0390-Bar-KOfhp, Figure S2: Construction of the vector pCAMBIA0390-Ben-Comfhp, Figure S3: Schematic diagram for preparing split-marker fragments and deletion of the Cmfhp gene.

Author Contributions: Conceptualization, J.-F.L. and L.-Q.G.; methodology, Y.Z. and B.-X.C.; data curation, Y.-H.Y.; writing-original draft preparation, H.-W.L.; writing—review and editing, H.-W.L. and Z.-W.Y.; visualization, H.-B.T.; project administration, J.-F.L. and L.-Q.G.; funding acquisition, J.-F.L., L.-Q.G., and Z.-W.Y. All authors have read and agreed to the published version of the manuscript.

Funding: This study was funded by the Key Realm R\&D Program of Guangdong Province (2018B020205001 and 2018B020205003), the National Natural Science Foundation of China (31572178, 31772373, and 31801918), the Construction Program of Innovation Team of Edible Fungus Industry Technology System of Guangdong Province (2019KJ103), and the Graduate Students Overseas Joint Education Program of South China Agricultural University (2018LHPY016).

Conflicts of Interest: The authors declare no conflict of interest.

\section{References}

1. Deng, L.; Zhou, T.Y.; Pi, L.; Zhao, X.H.; Han, T.; Li, Y.K.; Han, F. Optimization of microwave-assisted extraction of cordycepic acid and cordycepin from cultured Cordyceps militaris by response surface methodology. Asian J. Chem. 2013, 25, 8065-8071. [CrossRef] 
2. Nallathamby, N.; Guan-Serm, L.; Vidyadaran, S.; Abd Malek, N.; Raman, J.; Sabaratnam, V. Ergosterol of Cordyceps militaris attenuates LPS induced inflammation in BV2 microglia cells. Nat. Prod. Commun. 2015, 10, 885-886. [CrossRef] [PubMed]

3. Yong, T.Q.; Chen, S.D.; Xie, Y.Z.; Chen, D.L.; Su, J.Y.; Shuai, O.; Jiao, C.W.; Zuo, D. Cordycepin, a characteristic bioactive constituent in Cordyceps militaris, ameliorates hyperuricemia through URAT1 in hyperuricemic mice. Front. Microbiol. 2018, 9, 58. [CrossRef] [PubMed]

4. Zheng, Q.W.; Wei, T.; Yin, L.; Ye, Z.W.; Lin, J.F.; Guo, L.Q.; Yun, F.; Kang, L.Z. Developing a novel two-stage process for carotenoid production by Cordyceps militaris (Ascomycetes). Int. J. Med. Mushrooms 2019, 21, 47-57. [CrossRef] [PubMed]

5. Chiu, C.P.; Liu, S.C.; Tang, C.H.; Chan, Y.; El-Shazly, M.; Lee, C.L.; Du, Y.C.; Wu, T.Y.; Chang, F.R.; Wu, Y.C. Anti-inflammatory cerebrosides from cultivated Cordyceps militaris. J. Agric. Food Chem. 2016, 64, 1540-1548. [CrossRef]

6. Nurmamat, E.; Xiao, H.X.; Zhang, Y.; Jiao, Z.W. Effects of different temperatures on the chemical structure and antitumor activities of polysaccharides from Cordyceps militaris. Polymers 2018, 10, 430. [CrossRef]

7. Joshi, M.; Sagar, A.; Kanwar, S.S.; Singh, S. Anticancer, antibacterial and antioxidant activities of Cordyceps militaris. Indian J. Exp. Biol. 2019, 57, 15-20.

8. Dong, J.Z.; Wang, S.H.; Ai, X.R.; Yao, L.; Sun, Z.W.; Lei, C.; Wang, Y.; Wang, Q. Composition and characterization of cordyxanthins from Cordyceps militaris fruit bodies. J. Funct. Foods 2013, 5, 1450-1455. [CrossRef]

9. Xia, Y.L.; Luo, F.F.; Shang, Y.F.; Chen, P.L.; Lu, Y.Z.; Wang, C.S. Fungal cordycepin biosynthesis is coupled with the production of the safeguard molecule pentostatin. Cell Chem. Biol. 2017, 24, 1479-1489. [CrossRef]

10. Yang, T.; Sun, J.D.; Lian, T.T.; Wang, W.Z.; Dong, C.H. Process optimization for extraction of carotenoids from medicinal caterpillar fungus, Cordyceps militaris (Ascomycetes). Int. J. Med. Mushrooms 2014, 16, 125-135. [CrossRef]

11. Dong, J.Z.; Ding, J.; Yu, P.Z.; Lei, C.; Zheng, X.J.; Wang, Y. Composition and distribution of the main active components in selenium-enriched fruit bodies of Cordyceps militaris link. Food Chem. 2013, 137, $164-167$. [CrossRef] [PubMed]

12. Zhang, J.J.; Wang, F.; Liu, K.B.; Liu, Q.; Yang, Y.; Dong, C.H. Heat and light stresses affect metabolite production in the fruit body of the medicinal mushroom Cordyceps militaris. Appl. Microbiol. Biotechnol. 2018, 102, 4523-4533. [CrossRef]

13. Wang, F.; Liu, Q.; Zhang, J.J.; Liu, K.B.; Li, K.; Liu, G.J.; Dong, C.H. Comparative transcriptome analysis between a spontaneous albino mutant and its sibling strain of Cordyceps militaris in response to light stress. Front. Microbiol. 2018, 9, 1237. [CrossRef] [PubMed]

14. Jiang, K.Q.; Han, R.C. Rhf1 gene is involved in the fruiting body production of Cordyceps militaris fungus. J. Ind. Microbiol. Biotechnol. 2015, 42, 1183-1196. [CrossRef] [PubMed]

15. Shao, Y.; Tang, J.; Chen, S.L.; Wu, Y.H.; Wang, K.; Ma, B.; Zhou, Q.M.; Chen, A.H.; Wang, Y.L. milR4 and milR16 mediated fruiting body development in the medicinal fungus Cordyceps militaris. Front. Microbiol. 2019, 10, 83. [CrossRef] [PubMed]

16. Sun, S.J.; Deng, C.H.; Zhang, L.Y.; Hu, K.I. Molecular analysis and biochemical characteristics of degenerated strains of Cordyceps militaris. Arch. Microbiol. 2017, 199, 939-944. [CrossRef] [PubMed]

17. Yin, J.; Xin, X.D.; Weng, Y.J.; Gui, Z.Z. Transcriptome-wide analysis reveals the progress of Cordyceps militaris subculture degeneration. PLOS ONE 2017, 12, e0186279. [CrossRef]

18. Yang, T.; Guo, M.M.; Yang, H.J.; Guo, S.P.; Dong, C.H. The blue-light receptor CmWC-1 mediates fruit body development and secondary metabolism in Cordyceps militaris. Appl. Microbiol. Biotechnol. 2016, 100, 743-755. [CrossRef]

19. Lou, H.W.; Zhao, Y.; Tang, H.B.; Ye, Z.W.; Wei, T.; Lin, J.F.; Guo, L.Q. Transcriptome analysis of Cordyceps militaris reveals genes associated with carotenoid synthesis and identification of the function of the Cmtns gene. Front. Microbiol. 2019, 10, 2105. [CrossRef]

20. Canovas, D.; Marcos, J.F.; Marcos, A.T.; Strauss, J. Nitric oxide in fungi: Is there NO light at the end of the tunnel? Curr. Genet. 2016, 62, 513-518. [CrossRef]

21. Pengkit, A.; Jeon, S.S.; Son, S.J.; Shin, J.H.; Baik, K.Y.; Choi, E.H.; Park, G. Identification and functional analysis of endogenous nitric oxide in a filamentous fungus. Sci. Rep. 2016, 6, 30037. [CrossRef] [PubMed] 
22. Ollesch, G.; Kaunzinger, A.; Juchelka, D.; Schubert-Zsilavecz, M.; Ermler, U. Phospholipid bound to the flavohemoprotein from Alcaligenes eutrophus. Eur. J. Biochem. 1999, 262, 396-405. [CrossRef] [PubMed]

23. Lou, H.W.; Ye, Z.W.; Yun, F.; Lin, J.F.; Guo, L.Q.; Chen, B.X.; Mu, Z.X. Targeted gene deletion in Cordyceps militaris using the split-marker approach. Mol. Biotechnol. 2018, 60, 380-385. [CrossRef] [PubMed]

24. Lou, H.W.; Ye, Z.W.; Yu, Y.H.; Lin, J.F.; Guo, L.Q.; Chen, B.X.; Tang, H.B.; Wei, T.; Chen, L.T.; Yun, F. The efficient genetic transformation of Cordyceps militaris by using mononuclear protoplasts. Sci. Hortic. 2019, 243, 307-313. [CrossRef]

25. Lian, T.T.; Yang, T.; Liu, G.J.; Sun, J.D.; Dong, C.H. Reliable reference gene selection for Cordyceps militaris gene expression studies under different developmental stages and media. FEMS Microbiol. Lett. 2014, 356, 97-104. [CrossRef]

26. Chen, B.X.; Wei, T.; Ye, Z.W.; Yun, F.; Kang, L.Z.; Tang, H.B.; Guo, L.Q.; Lin, J.F. Efficient CRISPR-Cas9 gene disruption system in edible-medicinal mushroom Cordyceps militaris. Front. Microbiol. 2018, 9, 1157. [CrossRef]

27. Livak, K.J.; Schmittgen, T.D. Analysis of relative gene expression data using real-time quantitative PCR and the $2^{-\Delta \Delta C T}$ method. Methods 2001, 25, 402-408. [CrossRef]

28. Zheng, Z.L.; Huang, C.H.; Cao, L.; Xie, C.H.; Han, R.H. Agrobacterium tumefaciens-mediated transformation as a tool for insertional mutagenesis in medicinal fungus Cordyceps militaris. Fungal Biol. 2011, 115, $265-274$. [CrossRef]

29. Xie, X.G.; Fu, W.Q.; Zhang, F.M.; Shi, X.M.; Zeng, Y.T.; Li, H.; Zhang, W.; Dai, C.C. The endophytic fungus Phomopsis liquidambari increases nodulation and $\mathrm{N}_{2}$ fixation in Arachis hypogaea by enhancing hydrogen peroxide and nitric oxide signalling. Microb. Ecol. 2017, 74, 427-440. [CrossRef]

30. Yang, T.; Dong, C.H. Photo morphogenesis and photo response of the blue-light receptor gene Cmwc-1 in different strains of Cordyceps militaris. FEMS Microbiol. Lett. 2014, 352, 190-197. [CrossRef]

31. Kroll, K.; Pahtz, V.; Hillmann, F.; Vaknin, Y.; Schmidt-Heck, W.; Roth, M.; Jacobsen, I.D.; Osherov, N.; Brakhage, A.A.; Kniemeyer, O. Identification of hypoxia-inducible target genes of Aspergillus fumigatus by transcriptome analysis reveals cellular respiration as an important contributor to hypoxic survival. Eukaryot. Cell 2014, 13, 1241-1253. [CrossRef] [PubMed]

32. Gardner, P.R.; Costantino, G.; Salzman, A.L. Constitutive and adaptive detoxification of nitric oxide in Escherichia coli. J. Biol. Chem. 1998, 273, 26528-26533. [CrossRef] [PubMed]

33. Zheng, P.; Xia, Y.; Xiao, G.; Xiong, C.; Hu, X.; Zhang, S.; Zheng, H.; Huang, Y.; Zhou, Y.; Wang, S.; et al. Genome sequence of the insect pathogenic fungus Cordyceps militaris, a valued traditional Chinese medicine. Genome Biol. 2011, 12, R116. [CrossRef] [PubMed]

34. Zhang, G.Z.; Liang, Y. Improvement of fruiting body production in Cordyceps militaris by molecular assessment. Arch. Microbiol. 2013, 195, 579-585. [CrossRef]

35. Lou, H.; Lin, J.; Guo, L.; Wang, X.; Tian, S.; Liu, C.; Zhao, Y.; Zhao, R. Advances in research on Cordyceps militaris degeneration. Appl. Microbiol. Biotechnol. 2019, 103, 7835-7841. [CrossRef]

36. Lian, T.T.; Dong, C.H.; Yang, T.; Sun, J.D. Three types of geranylgeranyl diphosphate synthases from the medicinal caterpillar fungus, Cordyceps militaris (Ascomycetes). Int. J. Med. Mushrooms 2014, 16, 115-124. [CrossRef]

37. Chang, H.L.; Hsu, Y.T.; Kang, C.Y.; Lee, T.M. Nitric oxide down-regulation of carotenoid synthesis and PSII activity in relation to very high light-induced singlet oxygen production and oxidative stress in Chlamydomonas reinhardtii. Plant Cell Physiol. 2013, 54, 1296-1315. [CrossRef]

38. Lazar, E.E.; Wills, R.B.H.; Ho, B.T.; Harris, A.M.; Spohr, L.J. Antifungal effect of gaseous nitric oxide on mycelium growth, sporulation and spore germination of the postharvest horticulture pathogens, Aspergillus niger, Monilinia fructicola and Penicillium italicum. Lett. Appl. Microbiol. 2008, 46, 688-692. [CrossRef]

39. Ninnemann, H.; Maier, J. Indications for the occurrence of nitric oxide synthases in fungi and plants and the involvement in photoconidiation of Neurospora crassa. Photochem. Photobiol. 1996, 64, 393-398. [CrossRef]

(C) 2020 by the authors. Licensee MDPI, Basel, Switzerland. This article is an open access article distributed under the terms and conditions of the Creative Commons Attribution (CC BY) license (http://creativecommons.org/licenses/by/4.0/). 